\title{
Nonadiabatic approach to dimerization gap and optical absorption coefficient of the Su-Schrieffer-Heeger model
}

\author{
Qin Wang, ${ }^{\mathrm{a}, \mathrm{b}}$, Hong Chen ${ }^{\mathrm{a}}$ and Hang Zheng ${ }^{\mathrm{b}}$ \\ ${ }^{a}$ Pohl Institute of Solid State Physics, Tongji University, \\ Shanghai 200092, People's Republic of China \\ ${ }^{\mathrm{b}}$ Department of Applied Physics, Shanghai Jiao Tong University, \\ Shanghai 200030, People's Republic of China
}

\begin{abstract}
An analytical nonadiabatic approach has been developed to study the dimerization gap and the optical absorption coefficient of the Su-Schrieffer-Heeger model where the electrons interact with dispersive quantum phonons. By investigating quantitatively the effects of quantum phonon fluctuations on the gap order and the optical responses in this system, we show that the dimerization gap is much more reduced by the quantum lattice fluctuations than the optical absorption coefficient is. The calculated optical absorption coefficient and the density of states do not have the inverse-square-root singularity, but have a peak above the gap edge and there exist a significant tail below the peak. The peak of optical absorption spectrum is not directly corresponding to the dimerized gap. Our results of the optical absorption coefficient agree well with those of the experiments in both the shape and the peak position of the optical absorption spectrum.
\end{abstract}

PACS number(s): 71.38.-k; 71.20.Rv; 78.20.Ci; 71.45.Lr 


\section{Introduction}

Since the pioneering work of Su, Schrieffer and Heeger (SSH) [1], the SSH model has been widely used to investigate the properties of conducting polymers, especially polyacetylene $\left[(\mathrm{CH})_{x}\right]$ [2]. At half-filling, this system, as many quasi-one-dimensional materials, exhibits an instability against a periodic lattice distortion due to the Peierls dynamics. Because of a twofold degenerate ground state, this system allows for the soliton excitation with an associated electronic state at the center of the energy gap [3, 4]. While considerable effort has been made to understand excitation energies of solitons and polarons in these compounds, it is theoretically and experimentally significant to develop an accepted theory for their optical absorption spectrum. The recent exploitations of this model in studying the properties of nanotubes [5, 6, 7] renew further interesting of theoretical studies on the intrinsic physics of this model. In the previous works on conducting polymers, along with the expanding of measurements, theoretical attempts have been made to study the energy gap [8], lowlying excitation [3, 4, 9], and optical response [3, 10] of this system. However, there exist discrepancies between theories and experiments. (i) Within the adiabatic approximation, the calculated optical absorption coefficient of the perfectly dimerized lattice has an inversesquare-root edge singularity at $\omega=2 \Delta$ and there is no absorption inside the gap. But the

observed optical absorption spectrum of polyacetylene is quite different [11, 12, 13]. The singularity is absent, and there is a significant tail below the maximum. (ii) The energy gap deduced from the absorption edge is smaller than the activation energy of the dc conductivity 114, 15.

In $(\mathrm{CH})_{x}$ the zero-point motion of the lattice $\delta u(=0.03$ [15]) is comparable to the amplitude of the lattice Peierls distortion $u_{0}(=0.03$ [16] or 0.035 [17]). Although it has been stated that the quantum lattice fluctuations cause a slightly small reduction of the dimerization order parameter [18, 19, 20], if $\delta u \sim u_{0}$, as was pointed out in Ref. [2], 15], one might wonder why there should be a clearly defined gap in the spectrum at all and expect the optical absorption spectrum to peak at the correspondingly renormalized gap edge. In nonadiabatic case, the phonon frequency $\omega_{\pi}$ is finite. Generally speaking, the nonadiabatic effect will suppress the state order parameters of the system [19]. As the density-of-states (DOS) of electrons is concerned, the results of adiabatic approximations also have inverse-square-root singularity at the gap edge. By considering the nonadiabatic effect, the singularity may disappear [21]. The influence of the phonon frequency on DOS, the optical-absorption spectrum, and other order parameters in the range from $\omega_{\pi}=0$ to $\omega_{\pi} \rightarrow \infty$ should be studied for understanding the physics of electron-phonon interactions in nonadiabatic case.

The theoretical analysis of the coupled electron-phonon system for all values of the ionic mass and the electron-phonon coupling constant is a very difficult problem, especially when the quantum lattice fluctuation and the lattice dynamic (quantum phonon) effects are taken 
into account. This problem has been investigated by using the Monte Carlo simulation [18, 19], perturbation calculation [22], Green's-function technique [10], renormalization group analysis [23, 24], variational method of the squeezed-polaron wave-function [25], etc. In a resent work [26], this problem was studied by considering the spinless Holstein model and obtained some interesting results. In this paper, by considering $\mathrm{SSH}$ model, the more realistic case with spin- $\frac{1}{2}$ electrons, we focus on the properties of the effects of quantum lattice fluctuations on the dimerization energy gap and the optical-responses of the system with the view of understanding the measurement evidences and of interpreting why and how the dimerization energy gap is smaller than the optical absorption gap. Our analyses show that at finite phonon frequency, the peak position of optical absorption spectrum is not directly corresponding to the dimerized gap. The dimerization gap is much more reduced by the quantum lattice fluctuations than the optical absorption coefficient is. The effects of quantum lattice fluctuations on the dimerization gap and on the optical gap are essentially different, especially when phonon frequency $\omega_{\pi}$ is small. The reason for this is discussed and the relationship between the peak position of optical absorption spectrum and the true dimerization gap is obtained quantitatively.

\section{Model}

We start from the SSH model [1]

$$
H=-\sum_{l, s}\left[t+\alpha\left(u_{l}-u_{l+1}\right)\right]\left(c_{l, s}^{\dagger} c_{l+1, s}+c_{l+1, s}^{\dagger} c_{l, s}\right)+\sum_{l}\left[\frac{1}{2 M} P_{l}^{2}+\frac{1}{2} K\left(u_{l}-u_{l+1}\right)^{2}\right] .
$$

The notations in it are as usual [2] (throughout this paper, we set $\hbar=k_{B}=1$ ).

In Hamiltonian (11) the operators of lattice modes, $u_{l}$ and $p_{l}$, can be expanded by the phonon creation and annihilation operators $b_{q}^{\dagger}$ and $b_{q}$, and after Fourier transformation to momentum space, the $H$ becomes

$$
H=\sum_{k, s} \epsilon_{k} c_{k, s}^{\dagger} c_{k, s}+\sum_{q} \omega_{q}\left(b_{q}^{\dagger} b_{q}+\frac{1}{2}\right)+\frac{1}{\sqrt{N}} \sum_{q, k, s} g(k+q, k)\left(b_{-q}^{\dagger}+b_{q}\right) c_{k+q, s}^{\dagger} c_{k, s},
$$

where $\epsilon_{k}=-2 t \cos k$ is the bare band structure, $N$ is the total number of sites. The phonon frequency

$$
\omega_{q}=\sqrt{(4 K / M) \sin ^{2} \frac{q}{2}}
$$

and the coupling function

$$
g(k+q, k)=i 2 \alpha \sqrt{1 /\left(2 M \omega_{q}\right)}[\sin (k+q)-\sin k] .
$$

In the SSH model, the electron couples to the difference between the phonon amplitudes on the two neighboring sites and the form of the lattice vibration energy leads the phonons to be dispersive. 
Among the models for one-dimensional systems the Holstein model [27] and SSH model are the two typical electron-phonon coupling Hamiltonian studied by many previous authors. The Holstein model is for the on-site electron-phonon interaction with dispersionless phonons coupled with electron density operator and has played a central role in the polaron problem, while the SSH model is for the on-bond electron-phonon interaction and has been successful in describing the essential physics of conducting polymers. Phonon dispersion and different structures of the coupling terms lead to important differences in the physics of the two models. For the Holstein model, although numerical simulation [28] has been performed in confirmation of the supposition that it is likely the Peierls gap is more reduced by the quantum lattice fluctuations than the dimerization or the charge-density-wave amplitude, in other words, the quantum lattice fluctuations have a much strong effect on the Peierls gap than on the amplitude of the Peierls distortion, due to the difficulty in preparing practicably pure sample of molecular crystal materials, few of observation data are available for the comparison with theoretical results. Other models somewhat intermediate between the SSH and Holstein models, such as the model for $M X$ chain materials [29] and the model for electron-libron coupling in Polyaniline [30], because of their complex forms with Coulomb interactions and steric potential accounting for the ring torsion angle, will make the presentation of our analysis to be more prolix and inexplicit. The enormous measurement data of optical absorption spectrum in conducting polymers and the relatively simple form of SSH model render it the favorable system for our investigation into the nonadiabatic effect on the dimerization gap and the optical absorption spectrum of the electron-phonon coupling systems. The previous researches on this problem in SSH model by using a static Gaussian-random potential method to model the lattice disorder field [15, 31] and a configurational-coordinate method to treat the anharmonic lattice fluctuations [32] were phenomenological theories and have not obtained the true Peierls dimerization gap. It is the purpose of this paper to develop an nonadiabatic approach to study analytically this problem simply from the model Hamiltonian.

\section{Effective Hamiltonian}

The electron-phonon coupling and the Peierls dimerization are two main respects in our studying on the SSH model. First, in order to take into account the electron-phonon coupling, an unitary transformation is applied to $H$,

$$
H^{\prime}=\exp (S) H \exp (-S),
$$

where the generator $S$ is

$$
S=\frac{1}{\sqrt{N}} \sum_{q, k, s} \frac{g(k+q, k)}{\omega_{q}} \delta(k+q, k)\left(b_{-q}^{\dagger}-b_{q}\right) c_{k+q, s}^{\dagger} c_{k, s} .
$$


Here, we introduce a function $\delta\left(k^{\prime}, k\right)$ which is a function of the energies of incoming and outgoing electrons in the electron-phonon scattering process and it's form will be determined later. We divide the original Hamiltonian (2) into $H=H^{0}+H^{1}$, where $H^{0}$ contains the first two terms of $H$ and $H^{1}$ the last term. Then the unitary transformation can be proceed order by order,

$$
H^{\prime}=H^{0}+H^{1}+\left[S, H^{0}\right]+\left[S, H^{1}\right]+\frac{1}{2}\left[S,\left[S, H^{0}\right]\right]+O\left(\alpha^{3}\right) .
$$

The first-order terms in $H^{\prime}$ are

$$
\begin{aligned}
H^{1}+\left[S, H^{0}\right]= & \frac{1}{\sqrt{N}} \sum_{q, k, s} g(k+q, k)\left(b_{-q}^{\dagger}+b_{q}\right) c_{k+q, s}^{\dagger} c_{k, s} \\
& -\frac{1}{\sqrt{N}} \sum_{q, k, s} g(k+q, k) \delta(k+q, k)\left(b_{-q}^{\dagger}+b_{q}\right) c_{k+q, s}^{\dagger} c_{k, s} \\
& +\frac{1}{\sqrt{N}} \sum_{q, k, s} \frac{g(k+q, k)}{\omega_{q}} \delta(k+q, k)\left(b_{-q}^{\dagger}-b_{q}\right)\left(\epsilon_{k}-\epsilon_{k+q}\right) c_{k+q, s}^{\dagger} c_{k, s} .
\end{aligned}
$$

Note that the ground state $\left|g_{0}\right\rangle$ of $H^{0}$, the non-interacting system, is a direct product of a filled fermi-sea $|\mathrm{FS}\rangle$ and a phonon vacuum state $|\mathrm{ph}, 0\rangle$ :

$$
\left|g_{0}\right\rangle=|\mathrm{FS}\rangle|\mathrm{ph}, 0\rangle \text {. }
$$

Applying the first-order terms on $\left|g_{0}\right\rangle$ we get

$$
\left(H^{1}+\left[S, H^{0}\right]\right)\left|g_{0}\right\rangle=\frac{1}{\sqrt{N}} \sum_{q, k, s} g b_{-q}^{\dagger} c_{k+q, s}^{\dagger} c_{k, s}\left[1-\delta(k+q, k)\left(1-\frac{\epsilon_{k}-\epsilon_{k+q}}{\omega_{q}}\right)\right]\left|g_{0}\right\rangle,
$$

since $b_{q}|\mathrm{ph}, 0\rangle=0$. As the band is half-filled the Fermi energy $\epsilon_{\mathrm{F}}=0$. Thus $c_{k+q, s}^{\dagger} c_{k, s}|\mathrm{FS}\rangle \neq$ 0 only if $\epsilon_{k+q} \geq 0$ and $\epsilon_{k} \leq 0$. So, we have

$$
\left(H^{1}+\left[S, H^{0}\right]\right)\left|g_{0}\right\rangle=0
$$

if we choose

$$
\delta(k+q, k)=1 /\left(1+\left|\epsilon_{k+q}-\epsilon_{k}\right| / \omega_{q}\right) .
$$

This is nothing but making the matrix element of $H^{1}+\left[S, H^{0}\right]$ between $\left|g_{0}\right\rangle$ and the lowestlying excited states vanishing. Thus the first-order terms, which are not exactly canceled after the transformation, are related to the higher-lying excited states and should be irrelevant under renormalization [21]. The second-order terms in $H^{\prime}$ can be collected as follows:

$$
\begin{aligned}
& {\left[S, H^{1}\right]+\frac{1}{2}\left[S,\left[S, H^{0}\right]\right] } \\
= & \frac{1}{2 N} \sum_{q, k, s} \sum_{q^{\prime}, k^{\prime}} \frac{g(k+q, k) g\left(k^{\prime}+q^{\prime}, k^{\prime}\right)}{\omega_{q}} \delta(k+q, k)\left[2-\delta\left(k^{\prime}+q^{\prime}, k^{\prime}\right)\right]
\end{aligned}
$$




$$
\begin{aligned}
& \times\left(b_{-q}^{\dagger}-b_{q}\right)\left(b_{-q^{\prime}}^{\dagger}+b_{q^{\prime}}\right)\left(c_{k+q, s}^{\dagger} c_{k^{\prime}, s} \delta_{k, k^{\prime}+q^{\prime}}-c_{k^{\prime}+q^{\prime}, s}^{\dagger} c_{k, s} \delta_{k^{\prime}, k+q}\right) \\
& +\frac{1}{2 N} \sum_{q, k, s} \sum_{q^{\prime}, k^{\prime}} \frac{g(k+q, k) g\left(k^{\prime}+q^{\prime}, k^{\prime}\right)}{\omega_{q} \omega_{q^{\prime}}} \delta(k+q, k) \delta\left(k^{\prime}+q^{\prime}, k^{\prime}\right)\left(\epsilon_{k+q}-\epsilon_{k}\right) \\
& \times\left(b_{-q}^{\dagger}-b_{q}\right)\left(b_{-q^{\prime}}^{\dagger}-b_{q^{\prime}}\right)\left(c_{k+q, s}^{\dagger} c_{k^{\prime}, s} \delta_{k, k^{\prime}+q^{\prime}}-c_{k^{\prime}+q^{\prime}, s}^{\dagger} c_{k, s} \delta_{k^{\prime}, k+q}\right) \\
& -\frac{1}{N} \sum_{q, k, s} \sum_{k^{\prime}, s^{\prime}} \frac{g(k+q, k) g\left(k^{\prime}-q, k^{\prime}\right)}{\omega_{q}} \delta(k+q, k)\left[2-\delta\left(k^{\prime}-q, k^{\prime}\right)\right] c_{k+q, s}^{\dagger} c_{k, s} c_{k^{\prime}-q, s^{\prime}}^{\dagger} c_{k^{\prime},}\left(l_{3}\right)
\end{aligned}
$$

$\delta_{k^{\prime}, k+q}$ is the Kronecker $\delta$ symbol. All terms of higher order than $\alpha^{2}$ will be omitted in the following treatment.

Second, for the dimerized state, owing to the spontaneous lattice distortion, the neighboring atoms move in opposite directions. To take into account the static phonon-staggered ordering, we make a displacement transformation to $H^{\prime}$

$$
H^{\prime \prime}=\exp (R) H^{\prime} \exp (-R)
$$

Here the generator of the displacement operator $\exp (R)$ is

$$
R=-\sum_{l}(-1)^{l} u_{0} \sqrt{\frac{M \omega_{\pi}}{2}}\left(b_{l}^{\dagger}-b_{l}\right)
$$

If the ground state of $H$ is $|g\rangle$, then the ground state of $H^{\prime \prime}$ is $\left|g^{\prime}\right\rangle:|g\rangle=e^{-S} e^{-R}\left|g^{\prime}\right\rangle$. We assume that for $\left|g^{\prime}\right\rangle$ the electrons and phonons can be decoupled: $\left|g^{\prime}\right\rangle \approx|\mathrm{e}\rangle|\mathrm{ph}, 0\rangle$, where $|\mathrm{e}\rangle$ is the ground state for electrons. After averaging $H^{\prime \prime}$ over the phonon vacuum state we get an effective Hamiltonian for the electrons,

$$
\begin{aligned}
H_{\mathrm{eff}}= & \left\langle\mathrm{ph}, 0\left|H^{\prime \prime}\right| \mathrm{ph}, 0\right\rangle \\
= & 2 N K u_{0}^{2}+\sum_{k, s} E_{0}(k) c_{k, s}^{\dagger} c_{k, s}+\sum_{k, s} i \Delta_{0}(k) c_{k, s}^{\dagger} c_{k-\pi, s} \\
& -\frac{1}{N} \sum_{q, k, s} \sum_{k^{\prime}, s^{\prime}} \frac{g(k+q, k) g\left(k^{\prime}-q, k^{\prime}\right)}{\omega_{q}} \delta(k+q, k)\left[2-\delta\left(k^{\prime}-q, k^{\prime}\right)\right] c_{k+q, s}^{\dagger} c_{k, s} c_{k^{\prime}-q, s^{\prime}}^{\dagger} c_{k(,, \infty, 6 .)}
\end{aligned}
$$

where

$$
\begin{aligned}
& E_{0}(k)=\epsilon_{k}-\frac{1}{N} \sum_{k^{\prime}} \frac{g\left(k^{\prime}, k\right) g\left(k, k^{\prime}\right)}{\omega_{k^{\prime}-k}^{2}} \delta\left(k^{\prime}, k\right) \delta\left(k, k^{\prime}\right)\left(\epsilon_{k}-\epsilon_{k^{\prime}}\right) \\
& \Delta_{0}(k)=4 \alpha u_{0} \sin k[1-\delta(k-\pi, k)] .
\end{aligned}
$$

We find by means of the variational principle that the dimerized lattice displacement ordering parameter is

$$
u_{0}=-\frac{\alpha}{K N} \sum_{k>0, s} i \sin k[1-\delta(k-\pi, k)]\left\langle\mathrm{e}\left|\left(c_{k, s}^{\dagger} c_{k-\pi, s}-c_{k-\pi, s}^{\dagger} c_{k, s}\right)\right| \mathrm{e}\right\rangle
$$


The last term in $H_{\text {eff }}$ is a four-fermion interaction. As we are dealing with a one-dimensional system, how to treat the four-fermion interaction is a difficult problem.

Note that in the adiabatic limit, where $\omega_{\pi}=0$, one has $\delta\left(k^{\prime}, k\right)=0$, and $H_{\text {eff }}$ goes back to the adiabatic mean-field Hamiltonian,

$$
H_{\mathrm{eff}}\left(\omega_{\pi}=0\right)=2 N K u_{0}^{2}+\sum_{k, s} \epsilon_{k} c_{k, s}^{\dagger} c_{k, s}+\sum_{k>0, s} i 4 \alpha u_{0} \sin k\left(c_{k, s}^{\dagger} c_{k-\pi, s}-c_{k-\pi, s}^{\dagger} c_{k, s}\right) .
$$

It can be diagonalized exactly by means of the Bogoliubov transformation. The energy-gap parameter and the phonon-staggered ordering parameter in this limit can be obtained as

$$
\begin{gathered}
\Delta(k)=4 \alpha u_{0} \sin k, \\
m_{p}=\frac{1}{N} \sum_{l}(-1)^{l}\left\langle u_{l}\right\rangle=u_{0} .
\end{gathered}
$$

Thus, we have the relation

$$
\Delta_{\mathrm{ad}}=4 \alpha m_{p},
$$

same as the results of previous works [2, 3, 19] in the adiabatic limit.

On the other hand, in the antiadiabatic limit, where $\omega_{\pi} \rightarrow \infty$, we have $u_{0}=0, \delta\left(k^{\prime}, k\right)=1$, and $H_{\text {eff }}$ becomes

$$
H_{\text {eff }}\left(\omega_{\pi} \rightarrow \infty\right)=\sum_{k, s} \epsilon_{k} c_{k, s}^{\dagger} c_{k, s}-\frac{1}{N} \sum_{q, k, s} \sum_{k^{\prime}, s^{\prime}} \frac{g(k+q, k) g\left(k^{\prime}-q, k^{\prime}\right)}{\omega_{q}} c_{k+q, s}^{\dagger} c_{k, s} c_{k^{\prime}-q, s^{\prime}}^{\dagger} c_{k^{\prime}, s^{\prime}} .
$$

Returning to the real space, this Hamiltonian is

$$
\begin{aligned}
H_{\mathrm{eff}}\left(\omega_{\pi} \rightarrow \infty\right) & =-t \sum_{l, s}\left(c_{l+1, s}^{\dagger} c_{l, s}+c_{l, s}^{\dagger} c_{l+1, s}\right) \\
& -\frac{\alpha^{2}}{2 K} \sum_{l, s, s^{\prime}}\left(c_{l, s}^{\dagger} c_{l+1, s}+c_{l+1, s}^{\dagger} c_{l, s}\right)\left(c_{l, s^{\prime}}^{\dagger} c_{l+1, s^{\prime}}+c_{l+1, s^{\prime}}^{\dagger} c_{l, s^{\prime}}\right)
\end{aligned}
$$

For the spinless case, the on-site electron-electron interaction disappears because of the Pauli principle and this leads the Hamiltonian to be

$$
H_{\mathrm{eff}}\left(\omega_{\pi} \rightarrow \infty\right)=-t \sum_{l}\left(c_{l}^{\dagger} c_{l+1}+c_{l+1}^{\dagger} c_{l}\right)+\frac{\alpha^{2}}{K} \sum_{l}\left(c_{l}^{\dagger} c_{l} c_{l+1}^{\dagger} c_{l+1}-c_{l}^{\dagger} c_{l}\right)
$$

same as the exact effective Hamiltonian obtained by writing the phonon part of the SSH model as a functional integral and integrating out the phonon degrees of freedom [19]. In this case, the electrons experience an effective repulsive force between nearest neighbors, and for $\alpha^{2} / 2 K t=1$ the system undergoes a transition to a charge-density-wave state. This also is equivalent to the antiferromagnetic $X X Z$ spin chain model (via a Jordan-Wigner 
transformation) with the phase transition taking place at the isotropic point [33]. Thus our effective Hamiltonian works well in both the $\omega_{\pi}=0$ and the $\omega_{\pi} \rightarrow \infty$ limits.

Now the total Hamiltonian can be written as $\tilde{H}=\tilde{H}_{0}+\tilde{H}_{1}$, where $\tilde{H}_{1}$ includes the terms which are zero after being averaged over the phonon vacuum state, and

$$
\tilde{H}_{0}=\sum_{q} \omega_{q}\left(b_{q}^{\dagger} b_{q}+\frac{1}{2}\right)+H_{\mathrm{eff}}
$$

Through above two unitary transformations, the Hamiltonian is divided into two parts: $\tilde{H}_{0}$ and $\tilde{H}_{1}$. We believe that the $\tilde{H}_{0}$ contains the main physics of the coupling system. This is supported by: (a) $H_{\text {eff }}$ [Eq. (16)] works well for the both limits $\omega_{\pi}=0$ and $\omega_{\pi} \rightarrow \infty$, even if the electron-phonon coupling is strong; (b) the effect of the first order terms in $H^{\prime \prime}$ is eliminated in the lowest order of perturbation by introducing a function $\delta\left(k^{\prime}, k\right)$; (c) the results for the general $\omega_{\pi}$ in the spinless case are compared quite well [21] with those of the Monte Carlo simulations 19.

\section{Analytical Approach}

Note that the four-fermion interaction term in Eq. (16) goes to zero as $\omega_{\pi} \rightarrow 0$ [see Eq. (20)]. Therefore, in the case of small $\omega_{\pi}$, this term can be treated as a perturbation, and the unperturbed Hamiltonian is

$$
H_{\mathrm{eff}}^{0}=2 N K u_{0}^{2}+\sum_{k, s} E_{0}(k) c_{k, s}^{\dagger} c_{k, s}+\sum_{k, s} i \Delta_{0}(k) c_{k, s}^{\dagger} c_{k-\pi, s} .
$$

The four-fermion term can be re-written as

$$
\begin{aligned}
H_{\mathrm{eff}}^{\prime}= & -\frac{1}{N} \sum_{q, k, s} \sum_{k^{\prime}, s^{\prime}} \frac{g(k+q, k) g\left(k^{\prime}-q, k^{\prime}\right)}{\omega_{q}} \delta(k+q, k)\left[2-\delta\left(k^{\prime}-q, k^{\prime}\right)\right] \\
& \times\left(c_{k+q, s}^{\dagger} c_{k, s}-c_{k+q-\pi, s}^{\dagger} c_{k-\pi, s}\right)\left(c_{k^{\prime}-q, s^{\prime}}^{\dagger} c_{k^{\prime}, s^{\prime}}-c_{k^{\prime}-q-\pi, s^{\prime}}^{\dagger} c_{k^{\prime}-\pi, s^{\prime}}\right) \\
& +\frac{1}{N} \sum_{q, k, s} \sum_{k^{\prime}, s^{\prime}} \frac{g(k+q, k-\pi) g\left(k^{\prime}-q, k^{\prime}-\pi\right)}{\omega_{q-\pi}} \delta(k+q, k-\pi)\left[2-\delta\left(k^{\prime}-q, k^{\prime}-\pi\right)\right] \\
& \times\left(c_{k+q, s}^{\dagger} c_{k-\pi, s} c_{k^{\prime}-q-\pi, s^{\prime}}^{\dagger} c_{k^{\prime}, s^{\prime}}+c_{k+q-\pi, s}^{\dagger} c_{k, s} c_{k^{\prime}-q, s^{\prime}}^{\dagger} c_{k^{\prime}-\pi, s^{\prime}}\right) \\
& -\frac{1}{N} \sum_{q, k, s} \sum_{k^{\prime}, s^{\prime}} \frac{g(k+q, k-\pi) g\left(k^{\prime}-q, k^{\prime}-\pi\right)}{\omega_{q-\pi}} \delta(k+q, k-\pi)\left[2-\delta\left(k^{\prime}-q, k^{\prime}-\pi\right)\right] \\
& \times\left(c_{k+q-\pi, s}^{\dagger} c_{k, s} c_{k^{\prime}-q-\pi, s^{\prime}}^{\dagger} c_{k^{\prime}, s^{\prime}}+c_{k+q, s}^{\dagger} c_{k-\pi, s} c_{k^{\prime}-q, s^{\prime}}^{\dagger} c_{k^{\prime}-\pi, s^{\prime}}^{\dagger}\right) .
\end{aligned}
$$

In these terms we have the constraints

$$
k>0, \quad k^{\prime}>0, \quad k+q>0, \quad k^{\prime}-q>0 .
$$


We can distinguish between different physical processes. The first term in Eq. (29) is the forward scattering one, the second the backward one, and the last the Umklapp one. We use the Green's function method to implement the perturbation treatment and it is more convenient to work within a two-component representation,

$$
\Psi_{k, s}=\left(\begin{array}{l}
c_{k, s} \\
c_{k-\pi, s}
\end{array}\right), \quad k>0
$$

Thus we have

$$
\left\{\begin{array}{l}
\Psi_{k, s}^{\dagger} \sigma_{z} \Psi_{k, s}=c_{k, s}^{\dagger} c_{k, s}-c_{k-\pi, s}^{\dagger} c_{k-\pi, s} \\
\Psi_{k, s}^{\dagger} \sigma_{x} \Psi_{k, s}=c_{k, s}^{\dagger} c_{k-\pi, s}+c_{k-\pi, s}^{\dagger} c_{k, s} \\
\Psi_{k, s}^{\dagger} i \sigma_{y} \Psi_{k, s}=c_{k, s}^{\dagger} c_{k-\pi, s}-c_{k-\pi, s}^{\dagger} c_{k, s}
\end{array}\right.
$$

and the Hamiltonian becomes

$$
H_{\mathrm{eff}}^{0}=2 N K u_{0}^{2}+\sum_{k, s} E_{0}(k) \Psi_{k, s}^{\dagger} \sigma_{z} \Psi_{k, s}-\sum_{k, s} \Delta_{0}(k) \Psi_{k, s}^{\dagger} \sigma_{y} \Psi_{k, s}
$$

and

$$
\begin{aligned}
H_{\mathrm{eff}}^{\prime}= & -\frac{1}{N} \sum_{q, k, s} \sum_{k^{\prime}, s^{\prime}} \frac{g(k+q, k) g\left(k^{\prime}-q, k^{\prime}\right)}{\omega_{q}} \delta(k+q, k)\left[2-\delta\left(k^{\prime}-q, k^{\prime}\right)\right] \\
& \times \Psi_{k+q, s}^{\dagger} \sigma_{z} \Psi_{k, s} \Psi_{k^{\prime}-q, s^{\prime}}^{\dagger} \sigma_{z} \Psi_{k^{\prime}, s^{\prime}} \\
& +\frac{1}{2 N} \sum_{q, k, s} \sum_{k^{\prime}, s^{\prime}} \frac{g(k+q, k-\pi) g\left(k^{\prime}-q, k^{\prime}-\pi\right)}{\omega_{q-\pi}} \delta(k+q, k-\pi)\left[2-\delta\left(k^{\prime}-q, k^{\prime}-\pi\right)\right] \\
& \times\left(\Psi_{k+q, s}^{\dagger} \sigma_{x} \Psi_{k, s} \Psi_{k^{\prime}-q, s^{\prime}}^{\dagger} \sigma_{x} \Psi_{k^{\prime}, s^{\prime}}-\Psi_{k+q, s}^{\dagger} i \sigma_{y} \Psi_{k, s} \Psi_{k^{\prime}-q, s^{\prime}}^{\dagger} i \sigma_{y} \Psi_{k^{\prime}, s^{\prime}}\right) \\
& -\frac{1}{2 N} \sum_{q, k, s} \sum_{k^{\prime}, s^{\prime}} \frac{g(k+q, k-\pi) g\left(k^{\prime}-q, k^{\prime}-\pi\right)}{\omega_{q-\pi}} \delta(k+q, k-\pi)\left[2-\delta\left(k^{\prime}-q, k^{\prime}-\pi\right)\right] \\
& \times\left(\Psi_{k+q, s}^{\dagger} \sigma_{x} \Psi_{k, s} \Psi_{k^{\prime}-q, s^{\prime}}^{\dagger} \sigma_{x} \Psi_{k^{\prime}, s^{\prime}}+\Psi_{k+q, s}^{\dagger} i \sigma_{y} \Psi_{k, s} \Psi_{k^{\prime}-q, s^{\prime}}^{\dagger} i \sigma_{y} \Psi_{k^{\prime}, s^{\prime}}\right) .
\end{aligned}
$$

$\sigma_{\beta}(\beta=x, y, z)$ is the Pauli matrix. The matrix Green's function is defined as (the temperature Green's function is used and at the end let $T \rightarrow 0$ )

$$
\begin{aligned}
G_{s, s^{\prime}}(k, \tau) & =-\left\langle T_{\tau} \Psi_{k, s}(\tau) \Psi_{k, s^{\prime}}^{\dagger}(0)\right\rangle \\
& =\frac{1}{\beta} \sum_{n} \exp \left(-i \omega_{n} \tau\right) G_{s, s^{\prime}}\left(k, \omega_{n}\right) .
\end{aligned}
$$

The Dyson equation is

$$
G_{s, s^{\prime}}\left(k, \omega_{n}\right)=G_{s, s^{\prime}}^{0}\left(k, \omega_{n}\right)+G_{s, \alpha}^{0}\left(k, \omega_{n}\right) \Sigma_{\alpha, \beta}^{*}\left(k, \omega_{n}\right) G_{\beta, s^{\prime}}\left(k, \omega_{n}\right)
$$

where

$$
G_{s, s^{\prime}}^{0}\left(k, \omega_{n}\right)=\delta_{s, s^{\prime}}\left\{i \omega_{n}-E_{0}(k) \sigma_{z}+\Delta_{0}(k) \sigma_{y}\right\}^{-1}
$$


is the unperturbed Green's function. The self-energy $\Sigma_{\alpha, \beta}^{*}\left(k, \omega_{n}\right)$ can be calculated by the perturbation theory,

$$
\begin{aligned}
\Sigma_{\alpha, \beta}^{*}\left(k, \omega_{n}\right)= & \frac{T}{N} \sum_{k^{\prime}>0} \sum_{m} \frac{g\left(k, k^{\prime}\right) g\left(k^{\prime}, k\right)}{\omega_{k^{\prime}-k}} \delta\left(k^{\prime}, k\right)\left[2-\delta\left(k, k^{\prime}\right)\right]\left\{G_{\alpha, \beta}^{0}\left(k^{\prime}, \omega_{m}\right)+\sigma_{z} G_{\alpha, \beta}^{0}\left(k^{\prime}, \omega_{m}\right) \sigma_{z}\right\} \\
& +\frac{T}{N} \sum_{k^{\prime}>0} \sum_{m} \frac{g\left(k, k^{\prime}-\pi\right) g\left(k^{\prime}, k-\pi\right)}{\omega_{k^{\prime}-k-\pi}} \delta\left(k^{\prime}, k-\pi\right)\left[2-\delta\left(k, k^{\prime}-\pi\right)\right] \\
& \times\left\{i \sigma_{y} G_{\alpha, \beta}^{0}\left(k^{\prime}, \omega_{m}\right) i \sigma_{y}-\sigma_{x} G_{\alpha, \beta}^{0}\left(k^{\prime}, \omega_{m}\right) \sigma_{x}\right\} \\
& -\frac{T}{N} \sum_{k^{\prime}} \sum_{m} \frac{g(k, k-\pi) g\left(k^{\prime}, k^{\prime}-\pi\right)}{\omega_{\pi}}\left[\delta(k, k-\pi)+\delta\left(k^{\prime}, k^{\prime}-\pi\right)-\delta(k, k-\pi) \delta\left(k^{\prime}, k^{\prime}-\pi\right)\right] \\
& \times\left\{T_{r}\left[i \sigma_{y} G_{\alpha, \beta}^{0}\left(k^{\prime}, \omega_{m}\right)\right] i \sigma_{y}+T_{r}\left[\sigma_{x} G_{\alpha, \beta}^{0}\left(k^{\prime}, \omega_{m}\right)\right] \sigma_{x}\right\} .
\end{aligned}
$$

In the theoretical analysis we have taken into account the fact that only the Umklapp scattering terms affect the gap, and the forward and backward scattering terms contribute nothing to the "charge" gap [34, 35]. From Eq. (37) one can get that $\Sigma_{\alpha, \beta}^{*}\left(k, \omega_{n}\right)$ is irrelative to $\omega_{n}$, therefore the spectrum structure of $G_{s, s^{\prime}}\left(k, \omega_{n}\right)$ should be

$$
G_{s, s^{\prime}}\left(k, \omega_{n}\right)=\delta_{s, s^{\prime}}\left\{i \omega_{n}-E(k) \sigma_{z}+\Delta(k) \sigma_{y}\right\}^{-1}
$$

From $G_{s, s^{\prime}}\left(k, \omega_{n}\right)$ the elementary excitation spectrum in the gapped state can be derived

$$
W(k)=\sqrt{E^{2}(k)+\Delta^{2}(k)} .
$$

The renormalized band function and the gap function are

$$
\begin{aligned}
& E(k)=E_{0}(k)-\frac{1}{N} \sum_{k^{\prime}} \frac{2 \alpha^{2}}{K} \cos ^{2}\left(\frac{k^{\prime}+k}{2}\right) \delta\left(k^{\prime}, k\right)\left[2-\delta\left(k^{\prime}, k\right)\right] \frac{E_{0}\left(k^{\prime}\right)}{\sqrt{E_{0}^{2}\left(k^{\prime}\right)+\Delta_{0}^{2}\left(k^{\prime}\right)}}, \\
& \Delta(k)=4 \alpha u_{0} \sin k[c-d \delta(k-\pi, k)] .
\end{aligned}
$$

Here

$$
\begin{aligned}
c & =1+\frac{1}{N} \sum_{k>0, s} \frac{\alpha^{2}}{K} \delta(k-\pi, k) \sin k \frac{\Delta_{0}(k)}{2 \alpha u_{0} \sqrt{E_{0}^{2}(k)+\Delta_{0}^{2}(k)}}, \\
d & =1-\frac{1}{N} \sum_{k>0, s} \frac{\alpha^{2}}{K}[1-\delta(k-\pi, k)] \sin k \frac{\Delta_{0}(k)}{2 \alpha u_{0} \sqrt{E_{0}^{2}(k)+\Delta_{0}^{2}(k)}} .
\end{aligned}
$$

The equation to determine $u_{0}$ is

$$
1=\frac{1}{N} \sum_{k>0, s} \frac{\alpha^{2}}{K}[1-\delta(k-\pi, k)] \sin k \frac{\Delta(k)}{\alpha u_{0} W(k)} .
$$

These are basic equations in our theory. 
The phonon-staggered ordering parameter

$$
\begin{aligned}
m_{p} & =\frac{1}{N} \sum_{l}(-1)^{l}\left\langle u_{l}\right\rangle \\
& =\frac{1}{N} \sum_{k>0, s} \frac{\alpha}{K} \sin k \frac{\Delta(k)}{W(k)} .
\end{aligned}
$$

If $\omega_{\pi}=0$ we have $\delta\left(k^{\prime}, k\right)=0$ and $c=1$, Eq. (41) becomes the same as that in the adiabatic theory $\Delta_{\text {ad }}=\Delta(\pi / 2)=4 \alpha u_{0}$. Comparing Eq. (41) with the energy gap in the adiabatic case, $\Delta=4 \alpha u_{0}$, we have the gap in the nonadiabatic case, $\Delta=\Delta(\pi / 2)=$ $4 \alpha u_{0}[c-d]$. This is the true gap in the excitation spectrum.

Fig. 1 shows the elementary excitation spectrum $W(k)$ as function of the wave vector in the case of $\alpha^{2} / K t=0.336$ for different phonon frequencies $\omega_{\pi} / t=0.001,0.01,0.1$, and 0.2 . The adiabatic limit result is also shown in dash-dot-dot line for comparison. Near the Fermi surface, the change of phonon frequency strongly affects the elementary excitation spectrum. The dimerization gap decreases as the phonon frequency increases and changes very sensitively to the change of phonon frequency, especially when phonon frequency is small. The dimerization gap is much more reduced by the quantum lattice fluctuations than other order parameters, for instance, the phonon-staggered ordering parameter. The reason for this will be discussed later. In the mean field approximation the Peierls distortion opens a gap $2 \Delta_{\mathrm{MF}}$ and $\Delta_{\mathrm{MF}}=4 \alpha m_{p}$. Our results indicate that this relation holds only in the adiabatic limit. The effect of the quantum lattice fluctuations on the dimerization gap can be seen clearly also in the mean electron occupation number

$$
\sum_{s}\left\langle c_{k, s}^{\dagger} c_{k, s}\right\rangle=1-\frac{E(k)}{W(k)},
$$

Fig. 2 shows the mean electron occupation number as function of the wave vector in the cases of $\alpha^{2} / K t=0.2$ with $\omega_{\pi} / t=0$, and $\alpha^{2} / K t=0.4$ with $\omega_{\pi} / t=0$ and 0.2 . The more the phonon frequency or the electron-phonon coupling increases the more the Fermi surface is smeared. The strongest effect of the quantum phonon fluctuation at the Fermi surface leads to the strongest modulation of the dimerization gap. When $\alpha=0$, the system becomes one for free electrons and there is no long-range order, and consequently, as shown in dashdot-dot line, the system has a clear Fermi surface at $k=\pi / 2$. When $\alpha>0$, the effective electron-electron interactions induced by the electron-phonon coupling correlate the electrons and, thereby, the excitation spectrum of the system becomes one of quasi-particle excitations superseding the single electron excitations in $\alpha=0$ case.

The density-of-states of fermions $\rho(\omega)$ is

$$
\rho(\omega)=\frac{1}{N} \sum_{k, s} \delta\left(\omega-\sqrt{E^{2}(k)+\Delta^{2}(k)}\right)
$$




$$
=\frac{1}{\pi}\left(\left.\frac{d}{d k} \sqrt{E^{2}(k)+\Delta^{2}(k)}\right|_{k=f(\omega)}\right)^{-1}
$$

where, $k=f(\omega)$ is the inverse function of $\omega=\sqrt{E^{2}(k)+\Delta^{2}(k)}$. Fig. 3 shows the calculated DOS of fermions for electron-phonon coupling $\alpha^{2} / K t=0.336$ with $\omega_{\pi} / t=0.001,0.01$, and 0.04, respectively. One can see that a nonzero DOS stars from the gap edge and, for small values of $\omega_{\pi}$, there is a peak above the gap edge with a significant tail between it and the true gap edge. As $\omega_{\pi}$ increases, the peak height of the DOS decreases, and the peak broadens and moves to lower photon energy which implies that the dimerization gap becomes narrower and the Fermi surface becomes more smear. The inverse-square-root singularity at the gap edge in the adiabatic case [3, 18] disappears. For comparison, the adiabatic $\left(\omega_{\pi}=0\right)$ result is also shown in the dash-dot line which has an inverse-square-root singularity at gap edge. A previous analytic treatment on the modification of DOS due to lattice fluctuation showed the move of the DOS peak, but there existed still the singularity [10]. Our results consist with the spectrum measurements in both the peak position and the line shape of DOS.

The mean phonon occupation number (MPON) is

$$
\frac{1}{N} \sum_{q}\left\langle b_{q}^{\dagger} b_{q}\right\rangle=-\frac{2 K}{\omega_{\pi}} u_{0}^{2}+\frac{1}{N} \sum_{k>0, s} \frac{4 \alpha u_{0}}{\omega_{\pi}} \sin k \frac{\Delta(k)}{W(k)}+\frac{1}{N^{2}} \sum_{k>0, s} \sum_{k^{\prime}>0}^{\pi / 2} \frac{4 \alpha^{2}}{K} \frac{\delta^{2}\left(k, k^{\prime}\right)}{\omega_{k^{\prime}-k}} \cos ^{2} \frac{k+k^{\prime}}{2} .
$$

Fig. 4 shows the dependences of MPON on the phonon frequency for $\alpha^{2} / K t=0.336,0.50$ and 0.81 , respectively. MPON decreases as the electron-phonon coupling decreases or the phonon frequency increases. For small $\omega_{\pi}$, MPON changes very sensitively to the phonon frequency, and when $\omega_{\pi}=0$, MPON is divergent. Therefore, in point of the MPON, adiabatic approach is corresponding to the divergence of MPON, while for realistic materials, MPON is finite or even small.

\section{Optical Absorption Coefficient}

The optical absorption has remained an important source of information about the excitation spectrum of $(\mathrm{CH})_{x}$ [32], and the measurements of it's optical properties have provided much of the experimental basis for theoretical studies [36]. In the pristine sample the absorption curve peaks at about $2 \mathrm{eV}$ and the peak is followed by a broad tail extending into the gap. To appreciate the nonadiabatic effects on the optical excitation spectrum of $(\mathrm{CH})_{x}$, we further calculate the optical absorption coefficient under nonadiabatic circumstance by adopting the SSH model. The optical absorption coefficient $\alpha(\omega)$ can be expressed by the retarded Green's function [37] as follows:

$$
\alpha(\omega)=-\frac{2}{\pi \omega} \operatorname{Im} K^{R}(\omega),
$$


where $K^{R}$ is defined as

$$
K^{R}(\omega)=-i \int_{-\infty}^{0} e^{-i \omega t} d t\langle g|[j(0) j(t)-j(t) j(0)]| g\rangle .
$$

Here $j$ is the current operator [38],

$$
\begin{aligned}
j & =\operatorname{iev}_{F} \sum_{l, s}\left(c_{l, s}^{\dagger} c_{l+1, s}-c_{l+1, s}^{\dagger} c_{l, s}\right) \\
& =J \sum_{k>0, s} \sin k \Psi_{k, s}^{\dagger} \sigma_{z} \Psi_{k, s},
\end{aligned}
$$

$v_{F}=2 a t, J=2 e v_{F}$, and $j(t)=\exp (i H t) j \exp (-i H t)$ is the form of $j$ in the Heisenberg representation. Because the averaging of $\tilde{H}_{1}$ over the phonon vacuum state is zero, in the ground state at zero temperature $\tilde{H}_{1}$ can be neglected. Then, by using the approximately decoupling $\left|g^{\prime}\right\rangle \approx\left|g_{0}^{\prime}\right\rangle$, the ground state of $\tilde{H}_{0}$, and $\tilde{H} \approx \tilde{H}_{0}$ in the calculation

$$
\begin{aligned}
\langle g|j(0) j(t)| g\rangle & =\left\langle g^{\prime}\left|\left[e^{(S+R)} j e^{-(S+R)}\right] e^{i \tilde{H} t}\left[e^{(S+R)} j e^{-(S+R)}\right] e^{-i \tilde{H} t}\right| g^{\prime}\right\rangle \\
& \approx\left\langle g_{0}^{\prime}\left|\left[e^{S} j e^{-S}\right] e^{i \tilde{H}_{0} t}\left[e^{S} j e^{-S}\right] e^{-i \tilde{H}_{0} t}\right| g_{0}^{\prime}\right\rangle
\end{aligned}
$$

we can get

$$
\begin{aligned}
K^{R}(\omega)= & \frac{J^{2}}{N} \sum_{k>0, s}\left(\frac{1}{\omega-2 W(k)+i 0^{+}}-\frac{1}{\omega+2 W(k)-i 0^{+}}\right) \\
& \times\left[\sin ^{2} k-\frac{2}{N} \sum_{k^{\prime}} \frac{g\left(k^{\prime}, k\right) g\left(k, k^{\prime}\right)}{\omega_{k-k^{\prime}}^{2}} \delta^{2}\left(k^{\prime}, k\right) \sin k\left(\sin k-\sin k^{\prime}\right)\right] \frac{\Delta^{2}(k)}{W^{2}(k)} \\
& +\frac{J^{2}}{N^{2}} \sum_{k>0, k^{\prime}, s}\left(\frac{1}{\omega-\omega_{k-k^{\prime}}-W(k)-W\left(k^{\prime}\right)+i 0^{+}}-\frac{1}{\omega+\omega_{k-k^{\prime}}+W(k)+W\left(k^{\prime}\right)-i 0^{+}}\right) \\
& \times \frac{g\left(k^{\prime}, k\right) g\left(k, k^{\prime}\right)}{\omega_{k-k^{\prime}}^{2}} \delta^{2}\left(k^{\prime}, k\right)\left(\sin k-\sin k^{\prime}\right)^{2}\left[1-\operatorname{sign} k^{\prime} \frac{E(k) E\left(k^{\prime}\right)+\Delta(k) \Delta\left(k^{\prime}\right)}{W(k) W\left(k^{\prime}\right)}\right] .
\end{aligned}
$$

Thus, the optical absorption coefficient

$$
\begin{aligned}
\alpha(\omega)= & \frac{2 J^{2}}{\omega N} \sum_{k>0, s} \delta[\omega-2 W(k)] \frac{\Delta^{2}(k)}{W^{2}(k)} \\
& \times\left[\sin ^{2} k-\frac{2}{N} \sum_{k^{\prime}} \frac{4 \alpha^{2}}{K} \cos ^{3} \frac{k+k^{\prime}}{2} \operatorname{sign}\left(\sin \frac{k-k^{\prime}}{2}\right) \frac{\omega_{\pi} \sin k}{\left(\omega_{\pi}+4 t\left|\sin \frac{k-k^{\prime}}{2}\right|\right)^{2}}\right] \\
& +\frac{4 J^{2}}{\omega N^{2}} \sum_{k>0, s} \sum_{k^{\prime}} \delta\left[\omega-\omega_{k-k^{\prime}}-W(k)-W\left(k^{\prime}\right)\right]\left[1-\operatorname{sign} k^{\prime} \frac{E(k) E\left(k^{\prime}\right)+\Delta(k) \Delta\left(k^{\prime}\right)}{W(k) W\left(k^{\prime}\right)}\right] \\
& \times \frac{8 \alpha^{2}}{K} \cos ^{4} \frac{k+k^{\prime}}{2}\left|\sin \frac{k-k^{\prime}}{2}\right| \frac{\omega_{\pi}}{\left(\omega_{\pi}+4 t\left|\sin \frac{k-k^{\prime}}{2}\right|\right)^{2}},
\end{aligned}
$$


and the $\omega$-integrated spectral-weight function

$$
S(\omega)=\int_{0}^{\omega} \alpha\left(\omega^{\prime}\right) d \omega^{\prime}
$$

In Fig. 5, we compare our calculation (solid line) with the observed optical absorption (solid circles) [13] of trans- $(\mathrm{CH})_{x}$ polymer chain. The optical absorption coefficient $\alpha(\omega)$ is normalized to its peak value $\alpha\left(\omega_{\mathrm{p}}\right)$ and the photon energy $\omega$ is in unit of its peak value $\omega_{\mathrm{p}}$ for convenience to comparison. Here we use the input parameters $\alpha=4.1 \mathrm{eV} / \AA, K=21 \mathrm{eV} / \AA^{2}$, $t=2.5 \mathrm{eV}$, same as those of $\mathrm{Su}$, Schrieffer and Heeger [1], and $\omega_{\pi} / t=0.0652$. One can see that the agreement between the experiment result and our calculation is quite good. The relationships between the changes of the optical absorption shape as well as its peak position and the different phonon frequencies can be seen clearly in Fig. 6. The parameter values used are: $\alpha^{2} / K t=0.35, K=21 \mathrm{eV} / \AA^{2}$, and $t=2.5 \mathrm{eV}$ with $\omega_{\pi} / t=0.002$ (solid line) and 0.04 (dash line). The calculated true dimerization gaps relating to these two sets of parameter values are marked by the arrows. The solid circles denote the experimental results obtained from analysis of the inelastic electron-scattering data [3]. One can see that the spectrum broadens but the peak height decreases and shifts to lower photon energy as $\omega_{\pi}$ increases. The inverse-square-root singularity at the gap edge in the adiabatic case [3, 37, 38] and in the other methods [10] disappears because of the nonadiabatic effect. For finite $\omega_{\pi}$, there exists a significant tail below the peak. This figure shows again the excellent consistency of the optical absorption coefficient between our calculation and the experiment result in not only the peak position but also the line shape. It should be pointed out that in our calculation of the optical absorption coefficient, the choice of the parameter value set for the solid line requires $\alpha=4.28 \mathrm{eV} /$ Åbeening a little bit larger than $\alpha=4.1 \mathrm{eV} /$ Åused in Ref. [1], which implies that in our theory, due to the nonadiabatic effect, the larger electron-phonon coupling is required than that in adiabatic approach to obtain the same optical excitation energy. In addition, in experiments the measurement of optical absorption spectrum could be affected by various factors, such as the impurity in samples, the finite measurement temperature, and the finite measurement resolution. These may make the measured absorption spectrum to be broadened and lead to the slight discrepancy between the experiment result and our calculation.

Taking the excellent agreement of our results with the experiment observations on the optical absorption coefficient as the check and verification of the effectiveness of our analytical method, we further study the difference between the dimerization gap and the optical excitation response energy of the trans- $(\mathrm{CH})_{x}$ polymer chain within the SSH model. The experiments of many quasi-one-dimensional materials showed that the energy gap deduced from the absorption edge is smaller than the activation energy of the dc conductivity [39], which, as was pointed out [14, 15], can not be explained in methods assuming a static lattice 
distortion. A Monte Carlo calculation also suggested that the optical gap can be much larger than the dimerization gap [40]. Our calculated peak position of optical absorption coefficient $\omega_{\mathrm{p}}$ and the true dimerization gap $2 \Delta=2 \Delta(\pi / 2)$ as functions of phonon frequency $\omega_{\pi}$ are illustrated in Fig. 7. One can see that for the finite phonon frequency, the peak position of optical absorption coefficient is not directly corresponding to the true dimerization gap. The dimerization energy gap is smaller than the optical gap. It is the most notable that there is a discontinuous drop in the dimerization gap once the phonon frequency changes, no matter how small it is, from zero to finite, though at the adiabatic limit the dimerization gap $2 \Delta(\pi / 2)=\omega_{\mathrm{p}}$. After the drop the dimerization gap and the peak position of the optical absorption coefficient decrease as the phonon frequency increases. The dimerization gap is much more reduced by the quantum lattice fluctuations than the optical gap is. The dimerization gap is the value of Eq. (41) at the Fermi point $k=\pi / 2$, where the quantum lattice fluctuations have the strongest effect, while the optical absorption coefficient is the integral [see Eq. (54)] over the all Brillouin zone and the effect of the fluctuations is gentled. We note that in our theory, in mathematical viewpoint, the difference of the dimerization gap between the $\omega_{\pi}=0$ and $\omega_{\pi}>0$ cases mainly comes from the functional form of the gap [see Eq. (41)]. Comparing it with that in the adiabatic limit, one can see that the subgap states come from the quantum lattice fluctuations, i.e., the second term in the square bracket of Eq. (41). Substituting $\omega_{\pi}=0$ into Eq. (54), we obtain the optical absorption coefficient in the adiabatic limit

$$
\alpha(\omega)=\left.\frac{8 J^{2}}{\pi \omega^{3}}\left(4 \alpha u_{0}\right)^{2} \sin ^{4} k\left[\frac{d \omega}{d k}\right]^{-1}\right|_{k=\phi(\omega)},
$$

where, $k=\phi(\omega)$ is the inverse function of $\omega=2 \sqrt{\epsilon_{k}^{2}+\left(4 \alpha u_{0} \sin k\right)^{2}}$. Obviously, $k=\pi / 2$ is a singular point of Eq. (56), and, at this point, $\omega_{\mathrm{p}}=2\left(4 \alpha u_{0}\right)=2 \Delta$. Thus we get the same relation as in the adiabatic approach methods assuming a static lattice distortion, but our result indicates that this relation holds only in the adiabatic limit. In nonadiabatic case, in our theory, the logarithmic singularity in the integration of Eq. (54) in the adiabatic case is removed by the factor $1-\delta(k-\pi, k)$ as long as $\omega_{\pi}$ is finite. The effects of quantum lattice fluctuations on the dimerization gap and on the optical gap are essentially different, especially when $\omega_{\pi}$ is small. Though some works ascribed the difference between the dimerization gap and the optical excitation response energy to the electron-electron interactions [13], our calculation reveals that the effects of the quantum phonon can lead the dimerization gap to be much smaller than the optical gap in electron-phonon coupling systems. Contrary to the statement mentioned in the beginning that the quantum lattice fluctuations cause a slightly small reduction of the dimerization order parameter [18, 19, 20], our analysis shows that the dimerization gap being much more reduced behaves very different from other order parameters. 


\section{Conclusions}

We have studied the dimerization gap and the optical absorption coefficient of the $\mathrm{Su}-$ Schrieffer-Heeger model by means of developing an analytical nonadiabatic approach. By investigating quantitatively the effects of quantum phonon fluctuations on the gap order and the optical responses in this system, we have shown that the dimerization gap is much more reduced by the quantum lattice fluctuations than the optical absorption coefficient is. The calculated optical absorption coefficient and the density of states do not have the inversesquare-root singularity, but have a peak above the gap edge and there exists a significant tail below the peak. The peak of optical absorption spectrum is not directly corresponding to the dimerized gap. Our results of the optical absorption coefficient agree well with those of the experiments in both the shape and the peak position of the optical absorption spectrum. When the phonon frequency $\omega_{\pi}>0$, there still exists a static dimerization of the lattice but the quantum lattice fluctuations (the zero-point lattice motion) play a very important role. Taking the SSH model as a typical example for electron-phonon coupling systems in this study, we show that the strongest effects of the quantum lattice fluctuations at the Fermi surface lead the dimerization gap to be much smaller than the optical gap in electron-phonon coupling systems. The difference between the true dimerization gap and the peak position of optical absorption spectrum is obtained quantitatively.

This work was supported by NSF of China.

\section{References}

[1] W.P. Su, J.R. Schrieffer, and A.J. Heeger, Phys. Rev. Lett. 42, 1698 (1979).

[2] A.J. Heeger, S. Kivelson, J.R. Schrieffer, and W.P. Su, Rev. Mod. Phys. 60, 781 (1988).

[3] W.P. Su, J.R. Schrieffer, and A.J. Heeger, Phys. Rev. B 22, 2099 (1980).

[4] M.J. Rice, Phys. Lett. A 71, 781 (1988).

[5] K. Harigaya, Phys. Rev. B 45, 12071 (1992).

[6] Y. Zou, Z.J. Wang, and W.Z. Li, J. Phys. Chem. Solids, 58, 1653 (1997); 1657 (1997).

[7] J. Ma, R.K. Yuan, Phys. Rev. B 57, 9343 (1998); X.G. Wan, J.J.Gong, and D.Y. Xing, Solid State Commun. 107, 791 (1998); J. Jiang, J.M. Dong, D.Y. Xing, Phys. Rev. B 62, 13209 (2000).

[8] P.M. Grant, and I. Batra, Solid State Commun. 29, 225 (1979); J. Ashkanazi, E. Ehrenfreund, Z. Vardeny, and A.O. Brafman, Mol. Cryst. Liq. Cryst. 117, 193 (1985). 
[9] H. Takayama, Y.R. Lin-Liu, and K. Maki, Phys. Rev. B 21, 2388 (1980).

[10] Z.B. Su, Y.X. Wang, and L. Yu, Commun. in Theor. Phys. 6, 313 (1986).

[11] N. Suzuki, M. Ozaki, S. Etemad, A.J. Heeger, and A.G. Macdiarmid, Phys. Rev. Lett. 45, 1209 (1980).

[12] B.R. Weinberger, C.B. Roxlo, S. Etemad, G.L. Baker, and J. Orenstein, Phys. Rev. Lett. 53, 86 (1984).

[13] W.K. Wu and S. Kivelson, Phys. Rev. B 33, 8546 (1986).

[14] D. Moses, J. Chen, A. Denenstein, A.J. Heeger, and A.G. Macdiarmid, Solid State Commun. 40, 1007 (1981).

[15] R.H. McKenzie and J.W. Wilkins, Phys. Rev. Lett. 69, 1085 (1992).

[16] C.R. Fincher, C.E. Chen, A.J. Heeger, A.G. Macdiarmid, and J.B. Hastings, Phys. Rev. Lett. 48, 100 (1981).

[17] C.S. Yannoni and T.C. Clarke, Phys. Rev. Lett. 51, 1191 (1983).

[18] W.P. Su, Solid State Commun. 42, 497 (1982).

[19] E. Fradkin and J.E. Hirsch, Phys. Rev. B 27, 1680 (1983).

[20] A. Takahashi, Phys. Rev. B 46, 11550 (1992).

[21] H. Zheng, Phys. Rev. B 56, 14414 (1997).

[22] M. Nakahara and K. Maki, Phys. Rev. B 25, 7789 (1982).

[23] L.G. Caron and C. Bourbonnais, Phys. Rev. B 29, 4230 (1984).

[24] D. Schmeltzer, J. Phys. C 18, L1103 (1985).

[25] H. Zheng, D. Feinberg, and M. Avignon, Phys. Rev. B 39, 9405 (1989); H. Zheng and S.Y. Zhu, Phys. Rev. B 53, 3107 (1996).

[26] Q. Wang, H. Zheng, and M. Avignon, Phys. Rev. B 63, 014305 (2001).

[27] T. Holstein, Ann. Phys. 8, 325 (1959).

[28] E. Jeckelmann, C. Zhang, and R. White, Phys. Rev. B 60, 7950 (1999).

[29] S. Ichinose, Solid State Commun. 50, 137 (1984); K. Nasu, Phys. Spc. Jpn. 50, 235 (1981); J.T. Gammel, A. Saxena, I. Batistic, and A.R. Bishop, Phys. Rev. B 45, 6408 (1992). 
[30] J.M. Ginder and A.J. Epstein, Phys. Rev. B 41, 10674 (1990); D. Baranowski, H. Büttner, and J. Voit, Phys. Rev. B 47, 15472 (1993).

[31] K. Kim, R.H. McKenzie and J.W. Wilkins, Phys. Rev. Lett. 71, 4015 (1993).

[32] J. Yu, H. Matsuoka, and W.P. Su, Phys. Rev. B 37, 10367 (1988); B. Friedman and W.P. Su, Phys. Rev. B 39, 5152 (1989).

[33] C.N. Yang and C.P. Yang, Phys. Rev. 150, 321; 327 (1966).

[34] J. Solyom, Adv. Phys. 28, 209 (1979).

[35] J. Voit, Rep. Pro. Phys. 58, 977 (1995).

[36] S. Etemad, A.J. Heeger, and A.G. Macdiarmid, Annu. Rev. Phys. Chem. 33, 443 (1982).

[37] K. Maki and M. Nakahara, Phys. Rev. B 23, 5005 (1981).

[38] S. Kivelson, T.K. Lee, Y.R. Lin-Liu, I. Peschel, and L. Yu, Phys. Rev. B 25, 4173 (1982).

[39] P. Brüesch, S. Strässler, and H.R. Zeller, Phys. Rev. B 12, $219(1975)\left[\mathrm{K}_{2} \mathrm{Pt}(\mathrm{CN})_{4} \mathrm{Br}_{0.3}\right]$; R.P. McCall, Phys. Rev. B 39, 7760 (1989) [TCNQ compounds]; G. Minton and J.W. Brill, Phys. Rev. B 36, 4254 (1987) [( $\left.\left.\mathrm{TaSe}_{4}\right)_{2}\right]$.

[40] J.E. Hirsch, Phys. Rev. Lett. 51, 296 (1983). 


\section{Figure Caption}

Fig. 1 The fermionic excitation spectrum $W(k)$ as function of the wave vector in the case of $\alpha^{2} / K t=0.336$ for different phonon frequencies $\omega_{\pi} / t=0.001,0.01,0.1$, and 0.2 . The adiabatic limit result is also shown in dash-dot-dot line for comparison.

Fig. 2 The mean electron occupation number as function of the wave vector in the cases of $\alpha^{2} / K t=0.2$ with $\omega_{\pi} / t=0$, and $\alpha^{2} / K t=0.4$ with $\omega_{\pi} / t=0$ and 0.2 . The result for $\alpha=0$ is shown in dash-dot-dot line.

Fig. 3 The calculated DOS of fermions for electron-phonon coupling $\alpha^{2} / K t=0.336$ with $\omega_{\pi} / t=0.001,0.01$, and 0.04 , respectively. For comparison, the adiabatic $\left(\omega_{\pi}=0\right)$ result is also shown in the dash-dot line.

Fig. 4 The dependences of MPON on the phonon frequency for $\alpha^{2} / K t=0.336,0.50$ and 0.81 , respectively.

Fig. 5 The comparison of our calculation (solid line) with the observed optical absorption (solid circles) of trans- $(\mathrm{CH})_{x}$ polymer chain. The optical absorption coefficient $\alpha(\omega)$ is normalized to its peak value $\alpha\left(\omega_{\mathrm{p}}\right)$ and the photon energy $\omega$ is in unit of its peak value $\left.\omega_{\mathrm{p}}\right)$ for convenience to comparison. The input parameters are $\alpha=4.1 \mathrm{eV} / \AA, K=21 \mathrm{eV} / \AA^{2}$, $t=2.5 \mathrm{eV}$, and $\omega_{\pi} / t=0.0652$.

Fig. 6 The relationships between the changes of the optical absorption shape as well as its peak position and the different phonon frequencies. The parameter values used are: $\alpha^{2} / K t=0.35, K=21 \mathrm{eV} / \AA^{2}$, and $t=2.5 \mathrm{eV}$ with $\omega_{\pi} / t=0.002$ (solid line) and 0.04 (dash line). The calculated true dimerization gaps relating to these two sets of parameter values are marked by the arrows. The solid circles denote the experimental results obtained from analysis of the inelastic electron-scattering data.

Fig. 7 The calculated peak position of optical absorption coefficient $\omega_{\mathrm{p}}$ and the true dimerization gap $2 \Delta=2 \Delta(\pi / 2)$ as functions of phonon frequency $\omega_{\pi}$. 


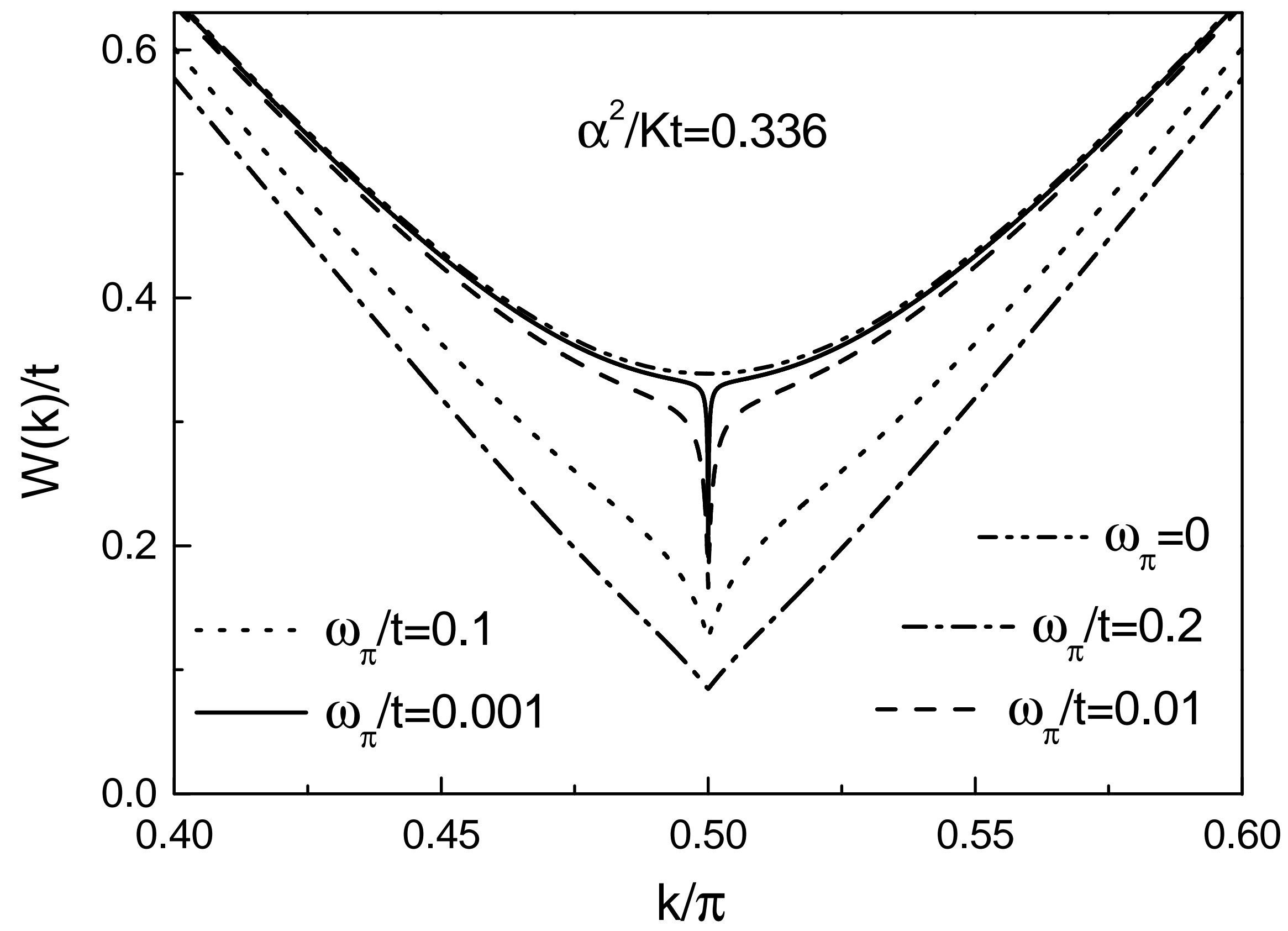




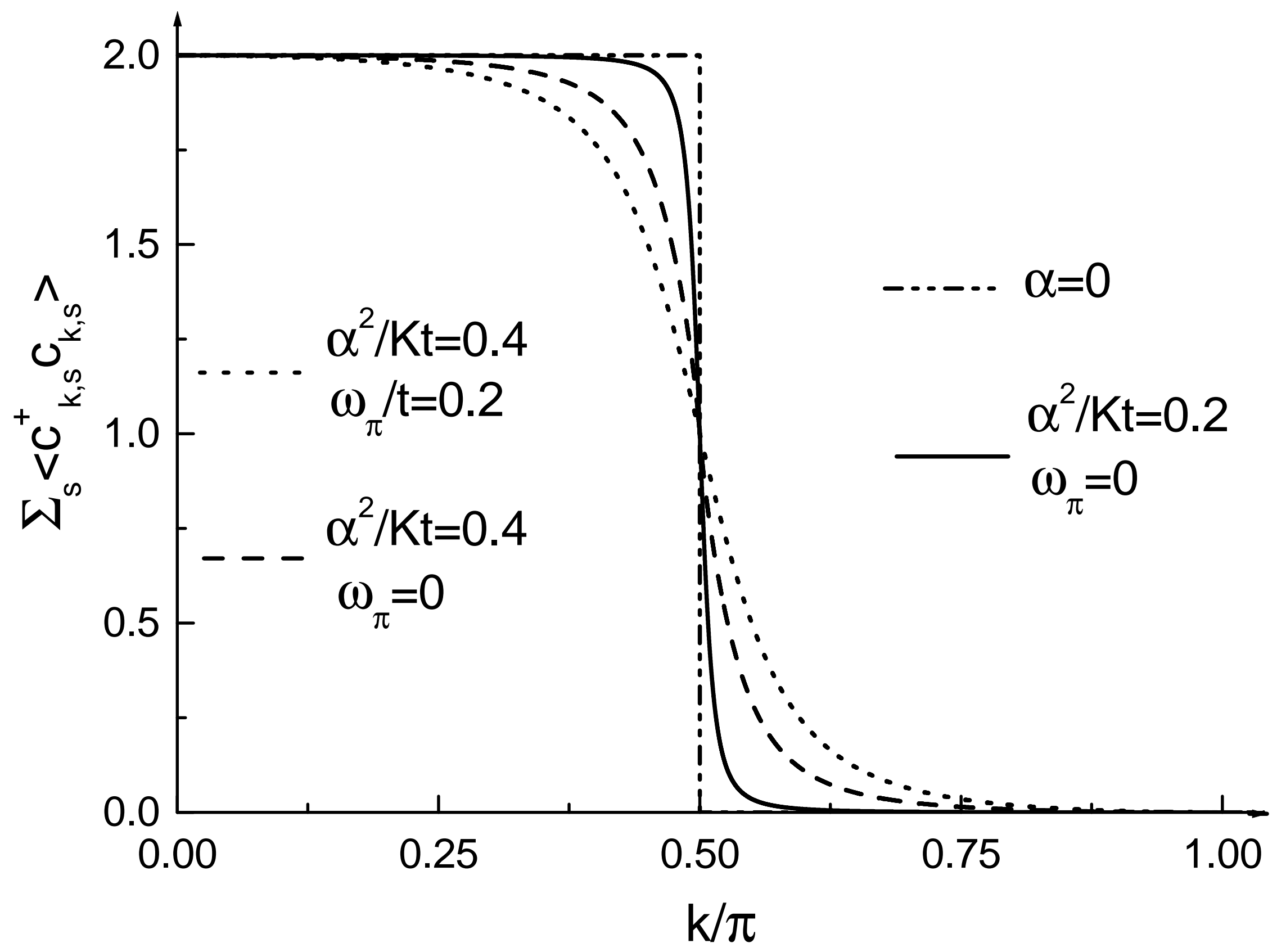




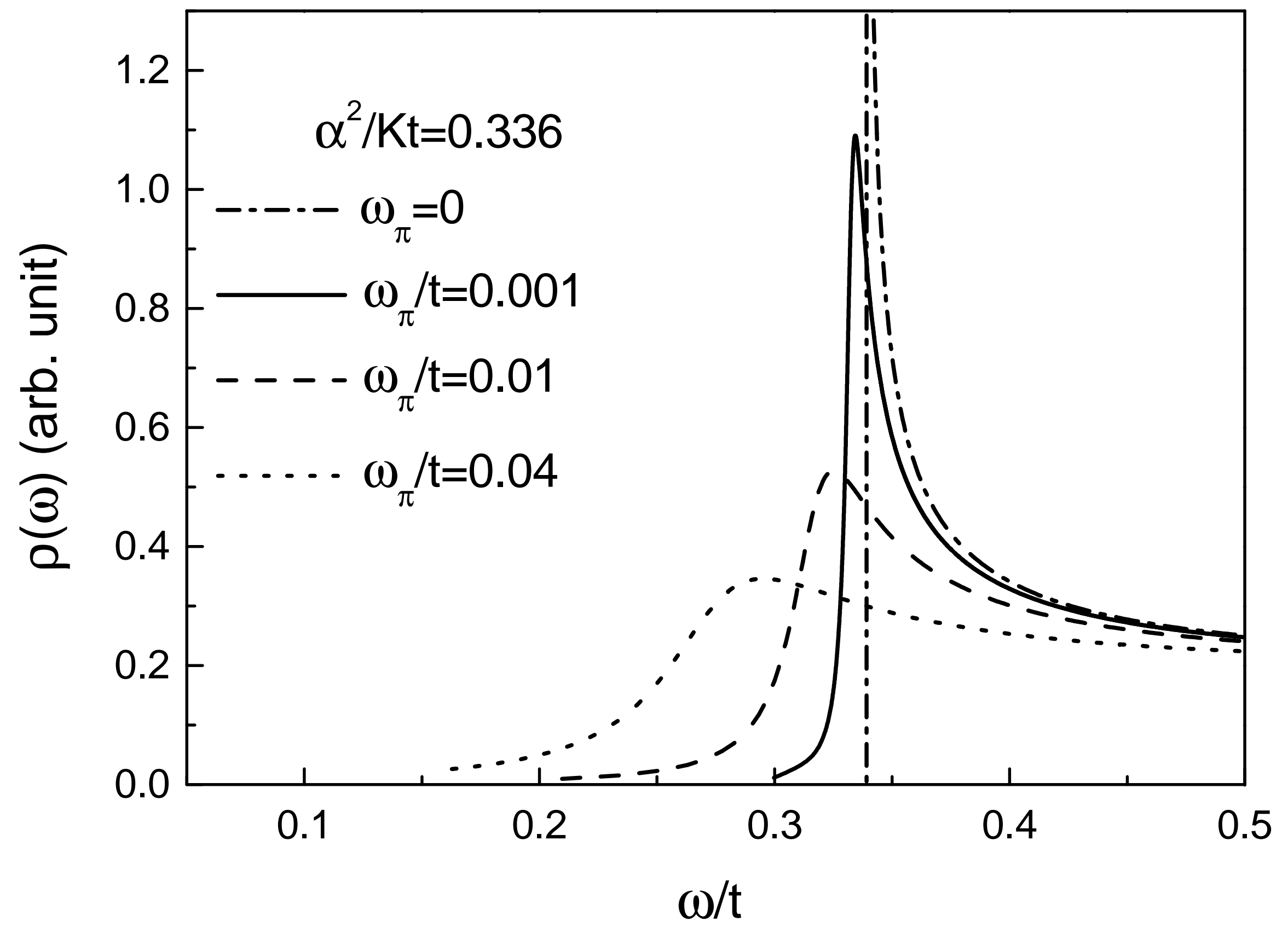




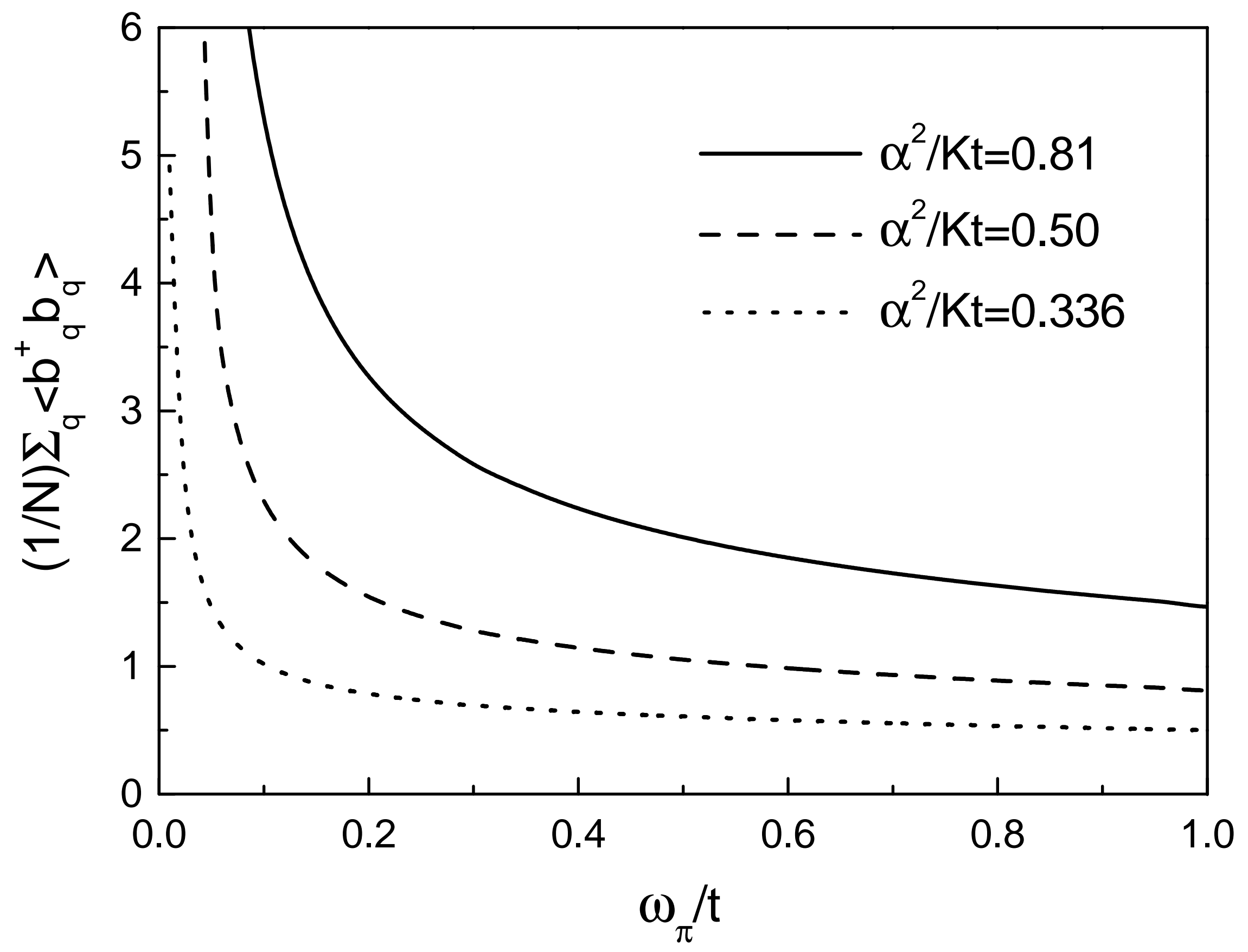




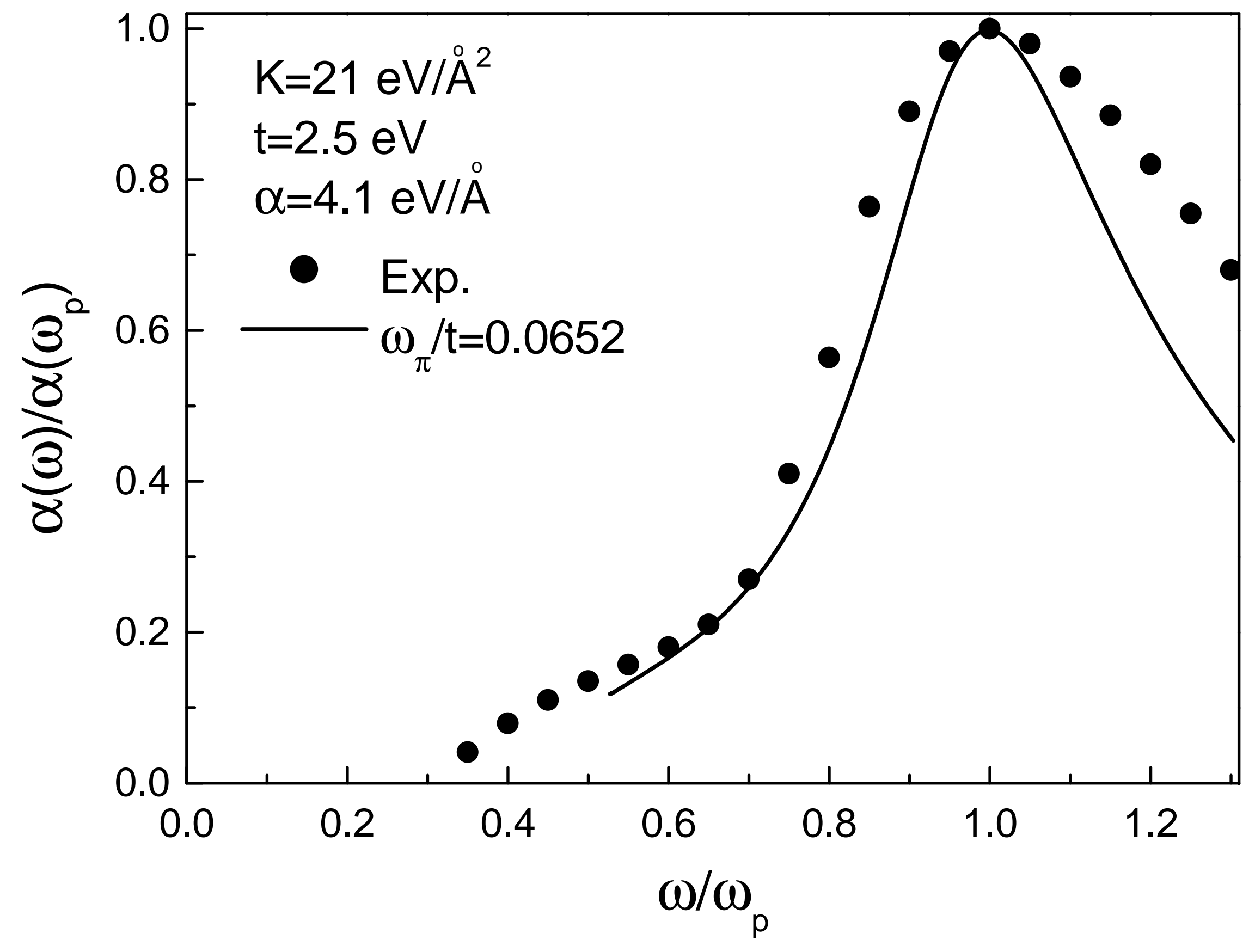




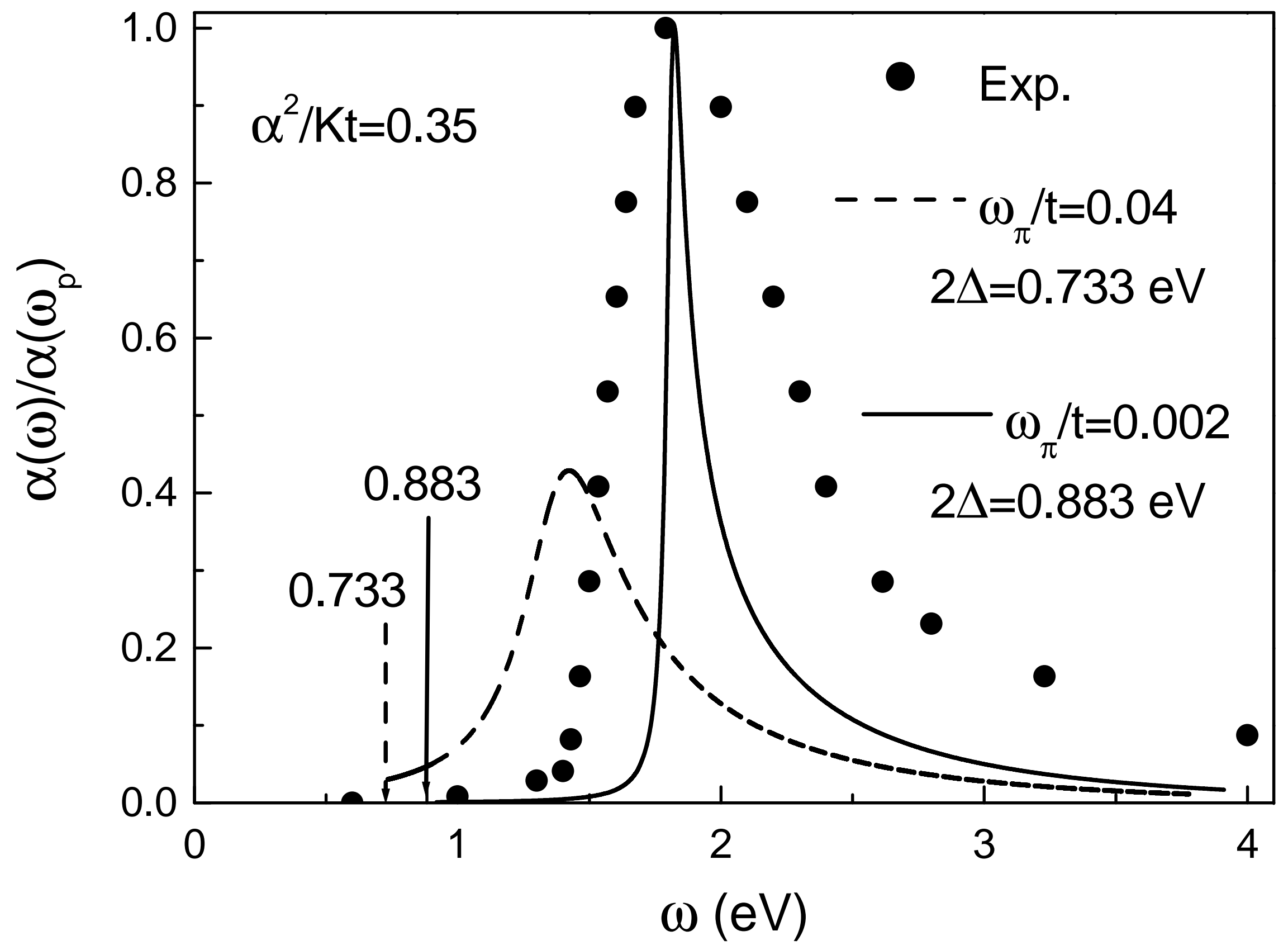




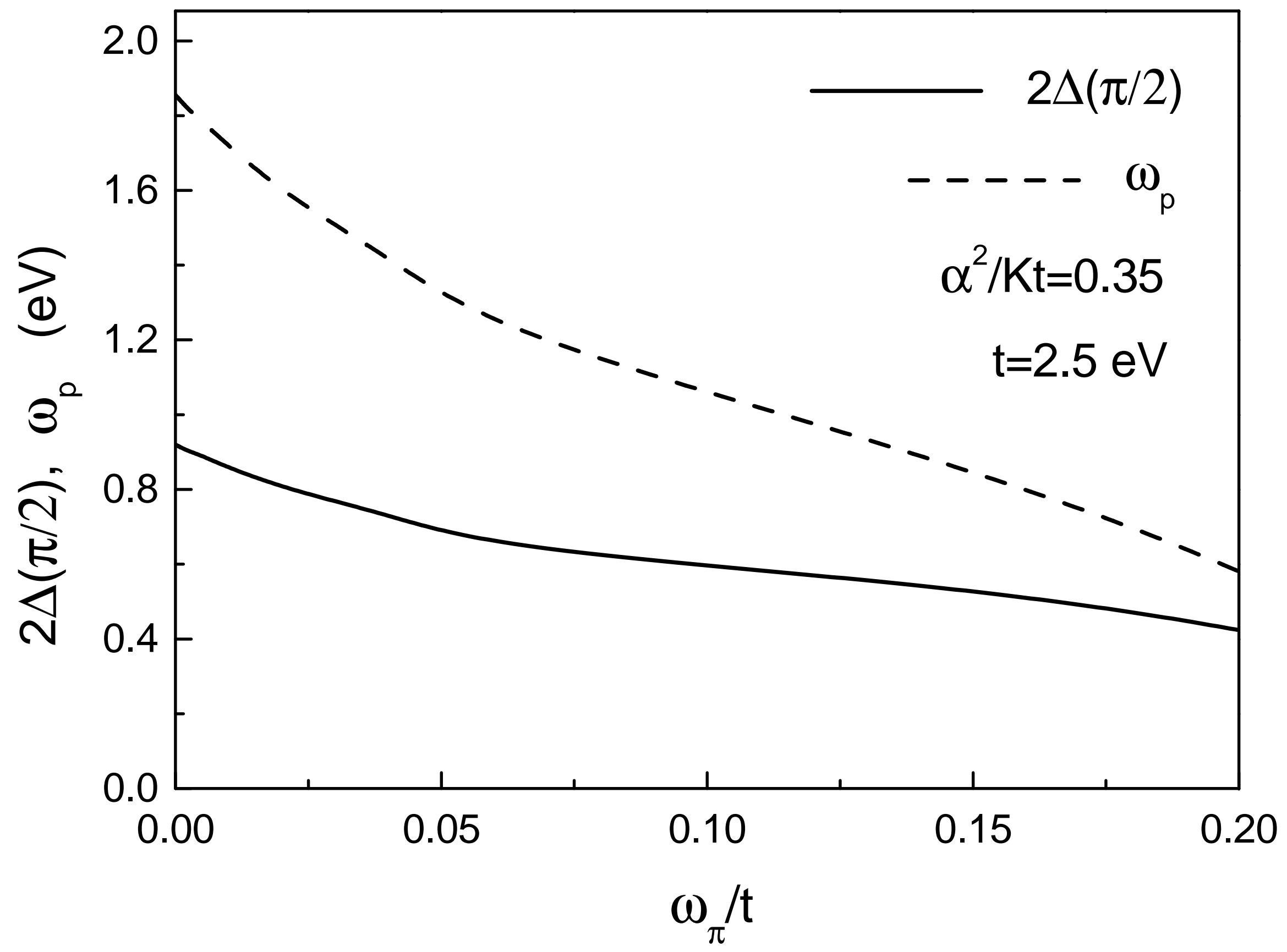

